Gut, 1987, 28, 1029-1032

\title{
Clinical trials
}

\section{Single dose tetracycline in cholera}

\author{
MOHAMMAD RAFIQUL ISLAM \\ From the International Centre for Diarrhoeal Disease Research, Dhaka, Bangladesh
}

\begin{abstract}
SUMMARY A randomised clinical trial was carried out to explore the efficacy of single dose tetracycline therapy in cholera. One hundred and eighteen adult patients were assigned to receive either tetracycline in a single $1 \mathrm{~g}$, or a single $2 \mathrm{~g}$ dose, or tetracycline $500 \mathrm{mg}$ every six hours four times, or no antibiotics as controls. The means of total liquid stool volumes after treatment were lower in the single $1 \mathrm{~g}$ dose group $(168.0 \pm 20.9 \mathrm{ml} / \mathrm{kg})$, in single $2 \mathrm{~g}$ dose group $(229.5 \pm 45.6 \mathrm{ml} / \mathrm{kg})$, and multiple dose group $(214 \pm 28.5 \mathrm{ml} / \mathrm{kg})$, than in the control group $(499.1 \pm 56.5 \mathrm{ml} / \mathrm{kg})$ $(\mathrm{p}<0 \cdot 05)$. Similarly, the means of durations of diarrhoea and intravenous fluid requirements were significantly lower in the single dose and multiple dose tetracycline groups, than in the controls $(\mathrm{p}<0 \cdot 05)$. The mean durations of excretion of Vibrio cholerae were significantly shortened from $3 \cdot 9 \pm 0 \cdot 2$ days in the control group to $1 \cdot 9 \pm 0 \cdot 2$ days in single $1 \mathrm{~g}$ dose, to $2 \cdot 2 \pm 0 \cdot 4$ days in single $2 \mathrm{~g}$ dose and $1 \cdot 3 \pm 0 \cdot 1$ days in multiple dose groups, respectively $(\mathrm{p}<0 \cdot 05)$. Three patients in the single $1 \mathrm{~g}$ dose group and two patients in single $2 \mathrm{~g}$ dose group had clinical relapses with excretion of $V$ cholerae during the relapses, but this was not significantly more frequent than that in the multiple dose group $(p>0.05)$. These findings suggest that although multiple dose tetracycline therapy remains the best choice, a single dose of either $1 \mathrm{~g}$ or $2 \mathrm{~g}$ tetracycline appears to be a reasonable alternative for the treatment of cholera as an adjunct to rehydration therapy.
\end{abstract}

Cholera is the severest of all forms of watery diarrhoeal diseases that may lead to dehydration and death. The effectiveness of tetracycline as an adjunct to rehydration therapy in the clinical management of acute cholera has been well documented. ${ }^{2}$ Tetracycline significantly shortens the period of diarrhoeal illness and reduces the volume of stool output and fluid requirements of patients with severe cholera. ${ }^{1-3}$ Treatment with tetracycline given for varying lengths of time $(1,2,3$, or 4 days) made no differences in clinical response. ${ }^{2}$ Also increasing the dose of tetracycline from two to three times did not appear to enhance its therapeutic effect. ${ }^{2}$ The most effective dose and duration of tetracycline therapy in cholera still remains unresolved. ${ }^{3}$ The advantages of single dose therapy include a saving of treatment cost, improved patients compliance and ease of administration, particularly when there is an epidemic.

Single dose doxycycline $(200 \mathrm{mg})$ although more expensive was found to be as effective as multiple

Address for correspondence: Diarrhoca Control Centre, Maternity and Children Hospital, Riyadh, PO Box 7855, Riyadh - 11117, KSA.

Received for publication 8 January 1987. dose therapy in cholera. ${ }^{+}$The objective of our study was to evaluate in a controlled trial, the clinical and bacteriological efficacy of three different treatment regimens, (a) a single dose of $1 \mathrm{~g}$ or (b) $2 \mathrm{~g}$ tetracycline and (c) a multiple dose of $2 \mathrm{~g}$ tetracycline in adult patients with severe cholera.

\section{Methods}

PATIENTS

The study was carried out at the International Centre for. Diarrhoeal Disease Research, Dhaka, Bangladesh. Adult patients who visited the hospital with a clinical history of acute watery diarrhoea of less than 24 hours duration with vibrios in faeces identified by dark field microscopy, ${ }^{5}$ evidence of dehydration according to WHO clinical criteria, ${ }^{6}$ and no antibiotic use within seven days before admission were selected for the study. The diagnosis of cholera was confirmed by bacteriologic identification of $V$ cholerae. Written informed consent was obtained. Patients were weighed and put on cholera cots designed to facilitate accurate separation of stool and urine. After a brief initial clinical assessment of the 
patient, intravenous rehydration was started using an isotonic solution containing $133 \mathrm{mmol}$ sodium, 13 mmol potassium, $98 \mathrm{mmol}$ chloride and $48 \mathrm{mmol}$ acetate per litre. Intravenous therapy was continued until diarrhoea stopped. Progress of hydration was assessed clinically by general appearance of the patient, good radial pulse, normal blood pressure, normal skin turgor, gain in body weight and good urination. Food was allowed as soon as the patients were able to eat.

Treatment was randomised among single $1 \mathrm{~g}$ dose, single $2 \mathrm{~g}$ dose, multiple dose or no tetracycline. Randomisation was done for each patient by drawing a slip labelled SD1, SD2, MD or O from an envelope initially filled with $50 \mathrm{SD} 1,25 \mathrm{SD} 2,25 \mathrm{MD}$, and 25 O's. Multiple dose therapy patients received $500 \mathrm{mg}$ tetracycline six hourly for four doses. Tetracycline was given orally.

Intravenous fluid intake and stool, vomitus and urine output were carefully measured every eight hours until diarrhoea stopped. 'End of diarrhoea' was defined to have occurred at the end of the last eight hour period in which liquid stool was passed. If a patient passed soft or formed stool thereby reaching 'end of diarrhoea' and subsequently passed liquid stool, this was considered to be a clinical relapse.

Catheter specimens of stool or rectal swabs were obtained daily for culture of $V$ cholerae $^{7}$ until cultures became negative for three consecutive days. Bacteria from colonies typical of $V$ cholerae were tested for agglutination with polyvalent 0 group 1 specific antisera and were tested for haemagglutinating activity with chicken erythrocytes.

If a patient become bacteriologically negative for at least two consecutive days and was subsequently positive for $V$ cholerae, this was considered to be bacteriological relapse.

After the end of the study, data of the four groups of patients were analysed by two tailed normal test ( $Z$ test), $\chi^{2}$ and Fisher exact test.

\section{Results}

The 118 patients were comparable in mean age, sex distribution, clinical dehydration, body weight and duration of diarrhoea before admission (Table 1). The majority of patients in each group were infected with Ogawa serotype, classical biotype, of $V$ cholerae. All 118 isolates of $V$ cholerae were susceptible to tetracycline by in vitro testing.

Patients treated with single and multiple doses of tetracycline responded quickly with sharp reductions in stool outputs in nearly all patients by the second eight hour period after treatment. Measurements of stool volumes at 16 hourly intervals after treatment showed that all three groups receiving tetracycline produced significantly less stool than the controls at $32,48,64$, and 80 hour after treatment $(p<0 \cdot 05$, Figure). The mean duration of diarrhoea of $35 \cdot 2 \mathrm{~h}$ after single $1 \mathrm{~g}$ dose, $42.8 \mathrm{~h}$ after single $2 \mathrm{~g}$ dose and $37.4 \mathrm{~h}$ after multiple dose treatment were significantly less than the mean of $85.4 \mathrm{~h}$ for the controls $(p<0.05)$ (Table 2). Similarly, the means of total stool output and intravenous fluid requirements in

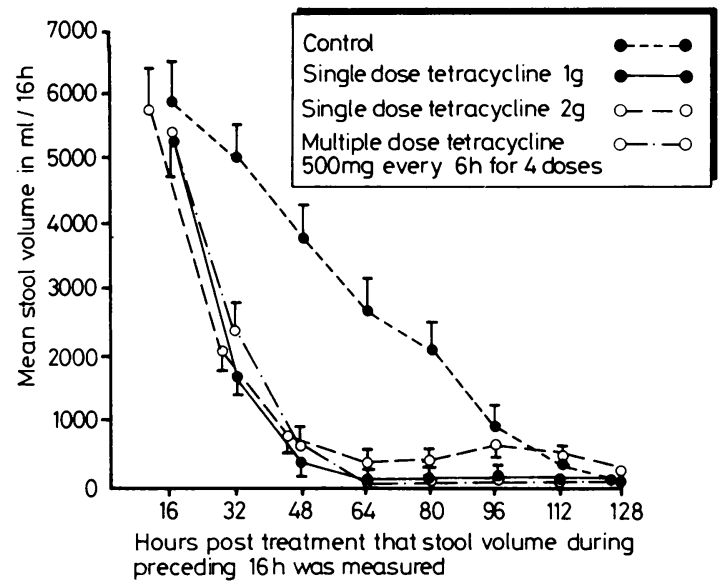

Figure Effect of therapy on stool output in cholera patients. Points are mean value of stool output during $16 \mathrm{~h}$ periods and brackets are I SEM unit. Mean values at times of 32, 48, 64, and 80 hour after treatment were significantly lower in patients treated with both single and multiple doses of tetracycline when compared with controls $(<0 \cdot 05)$.

Table 1 Comparison of the characteristics of cholera patients on admission treated with single $1 \mathrm{~g}$ or $2 \mathrm{~g}$ dose tetracycline, multiple dose tetracycline and controls without tetracycline. Values are means $\pm S E M$ or percentages of patients with the features

\begin{tabular}{|c|c|c|c|c|}
\hline Characteristics & Single I g dose $(n=45)$ & Single $2 \mathrm{~g}$ dose $(n=23)$ & Multiple dose $(n=25)$ & Controls $(n=25)$ \\
\hline Age in years & $30 \cdot 4 \pm 2 \cdot 1$ & $28 \cdot 4 \pm 2 \cdot 5$ & $26 \cdot 3 \pm 1 \cdot 9$ & $33 \cdot 2 \pm 3 \cdot 2$ \\
\hline Male/female & $30 / 15$ & $12 / 11$ & $10 / 15$ & $11 / 14$ \\
\hline Body weight in $\mathrm{kg}$ after rehydration & $42 \cdot 9 \pm 1 \cdot 1$ & $43 \cdot 6 \pm 2 \cdot 0$ & $40 \cdot 7 \pm 1 \cdot 4$ & $42 \cdot 5 \pm 1 \cdot 1$ \\
\hline Duration of diarrhoca before admission (h) & $13 \cdot 3 \pm 1 \cdot 1$ & $12 \cdot 1 \pm 1 \cdot 1$ & $13 \cdot 8 \pm 1 \cdot 2$ & $16 \cdot 4 \pm 1 \cdot 4$ \\
\hline \multicolumn{5}{|c|}{ Clinical dehydration: } \\
\hline Moderate & $22 \%$ & $20 \%$ & $12 \%$ & $12 \%$ \\
\hline
\end{tabular}


the single doses and multiple dose tetracycline groups were less than half of that of the controls. The mean durations of vibrio excretion in stool were 1.9 days in single $1 \mathrm{~g}$ dose, $2 \cdot 2$ days in single $2 \mathrm{~g}$ dose, $1 \cdot 3$ days in multiple dose but 3.9 days in control group $(\mathrm{p}<0.05)$. Increasing the single dose of tetracycline from $1 \mathrm{~g}$ to $2 \mathrm{~g}$ did not enhance its therapeutic efficacy (Table 2).

Clinical relapses were defined as the return of liquid stool after passing solid stool. Three patients in the single $1 \mathrm{~g}$ dose treatment group, two patients in the single $2 \mathrm{~g}$ dose group experienced clinical relapses and all had positive stool cultures for $V$ cholerae of the same biotype and serotype as they had in their initial episodes. These relapses occurred two to three days after the patients had ceased passing liquid stool and four of these patients required intravenous fluid therapy. No clinical relapses occurred in patients in the multiple dose and control groups. The incidences of clinical relapses in the single $1 \mathrm{~g}$ dose group of $3 / 45$ and $2 / 23$ in the single $2 \mathrm{~g}$ dose group, however, were not significantly greater than in the multiple dose group (Fisher's Exact Test).

The bacteriological responses were prompt in all single and multiple dose groups (Table 3 ). By day 2 , or just $24 \mathrm{~h}$ after the start of treatment, the rates of isolating $V$ cholerae from stool cultures were significantly less in three treatment groups than in the control group $(p<0 \cdot 05)$. At this time, the rate of $2 / 25$ isolations in the multiple dose groups was significantly less than $13 / 45$ in the single $1 \mathrm{~g}$ dose group $(p<0.05)$, and than $10 / 23$ in the single 2 g dose group $(p<0.05)$. Thereafter, the rates of isolation were similarly low in both treatment groups. Bacteriological relapses were defined as the isolation of $V$ cholerae from a patient who had negative cultures on two preceding days. There were seven bacteriological relapses in the single $1 \mathrm{~g}$ dose group, two relapses in the single $2 \mathrm{~g}$ dose group, and three relapses in the multiple dose group.

\section{Discussion}

Although emergence of multiply antibiotic resistant $V$ cholerae has been reported in few cases in Bangladesh $^{\times}$and Tanzania," tetracycline has remained the drug of choice in the treatment of cholera and it had been proved beyond doubt that it reduces gastrointestinal fluid loss, duration of diarrhoea and also eliminates $V$ cholerae from stool within 48 hours. Using single dose antibiotic therapy the physician can ensure that the patient has taken his full course of therapy and does not have to depend on patient compliance. The efficacy of single large dose therapy in infections in probably related to the rapid attainment of high concentration of the drug at the

Table 2 Duration of diarrhoea, output of stool, intravenous fluid requirement, and vibrio excretion of cholera patients treated with single dose tetracycline $1 \mathrm{~g}$ or $2 \mathrm{~g}$, multiple dose tetracycline $500 \mathrm{mg}$ every six $h$ four times, and controls not receiving antibiotic. Values are mean $\pm S E M^{*}$

\begin{tabular}{lllll}
\hline & $\begin{array}{l}\text { Duration of diarrhoea } \\
\text { after treatment }(h)\end{array}$ & $\begin{array}{l}\text { Total liquid stool output } \\
\text { in ml/kg during hospital } \\
\text { stay }\end{array}$ & $\begin{array}{l}\text { Intravenous fluid } \\
\text { requirement (ml/kg) }\end{array}$ & $\begin{array}{l}\text { Duration of positive } \\
\text { vibrio culture in stool } \\
\text { (day) }\end{array}$ \\
\hline Single 1 g dose tetracycline $(\mathrm{n}=45)$ & $35 \cdot 2 \pm 2 \cdot 8$ & $168 \cdot 0 \pm 20 \cdot 9$ & $202 \cdot 9 \pm 23 \cdot 6$ & $1 \cdot 9 \pm 0 \cdot 2$ \\
Single 2g dose tetracycline $(\mathrm{n}=23)$ & $42 \cdot 8 \pm 6 \cdot 7$ & $229 \cdot 5 \pm 45 \cdot 6$ & $260 \cdot 7 \pm 48 \cdot 9$ & $2 \cdot 2 \pm 0 \cdot 4$ \\
Multiple dose tetracycline $(\mathrm{n}=25)$ & $37 \cdot 4 \pm 3 \cdot 4$ & $214 \cdot 5 \pm 28 \cdot 5$ & $258 \cdot 3 \pm 29 \cdot 3$ & $1 \cdot 3 \pm 0 \cdot 4$ \\
Control $(\mathrm{n}=25)$ & $85 \cdot 4 \pm 5 \cdot 6$ & $499 \cdot 1 \pm 56 \cdot 5$ & $526 \cdot 8 \pm 63 \cdot 8$ & $3 \cdot 9 \pm 0 \cdot 2$ \\
\hline
\end{tabular}

* Means of duration of diarrhoea, total liquid stool output, intravenous fluid requirement, and duration of vibrio excretion for both single dose and multiple dose tetracycline were significantly lower than for controls ( $p>0.001$ by normal ' $Z$ ' test). Means of these determinations were not significantly different among single doses and multiple dose groups $(p>0 \cdot 05)$.

Table 3 Isolation of $\mathrm{V}$ cholerae from stools of patients treated with single dose tetracycline, multiple dose tetracycline and controls without tetracycline. Numbers are cases with positive culture on each day in hospital. R refers to number of bacteriological relapses, defined as 2 or more days of negative culture followed by a positive culture

\begin{tabular}{|c|c|c|c|c|c|c|c|}
\hline Treatment group & Day I & $\operatorname{Day} 2^{*}$ & Day 3+ & Day 4t & Day $5 \dagger$ & Day $6+$ & Day 7 \\
\hline Single $1 \mathrm{~g}$ dose & 45 & 13 & 7 & $5+5 R$ & $3+2 \mathrm{R}$ & 2 & 1 \\
\hline Single $2 \mathrm{~g}$ dose & 23 & 10 & 3 & $4+2 \mathrm{R}$ & 3 & 2 & 1 \\
\hline Control & 25 & 19 & 18 & $14+1 \mathrm{R}$ & $14+2 \mathrm{R}$ & $14+1 \mathrm{R}$ & () \\
\hline
\end{tabular}

*On day 2 , the frequency of positive cultures was significantly greater in controls than in cases treated with either single or multiple doses of tetracycline $\left(\mathrm{p}<0.05\right.$ by $\chi^{2}$ test $)$. The frequency of positive culture was significantly greater in cases treated with single doses than with multiple doses $\left(p<0.05\right.$ by $\chi^{2}$ test $)$; †On days $3-6$, the frequencies of positive cultures were greater in controls than in patients treated with either single or multiple doses of tetracycline. 
site of infections causing rapid destruction of the invading organisms.

Our study has shown that single $1 \mathrm{~g}$ or $2 \mathrm{~g}$ dose tetracycline was as effective as multiple dose therapy when mean values of duration of diarrhoea, liquid stool output, intravenous fluid requirement, and excretion of $V$ cholerae were compared. Although initial response is good, clinical relapses with excretion of $V$ cholerae during the relapses occurred in three patients $(6.6 \%)$ in the single $1 \mathrm{~g}$ dose group and two patients $(8.7 \%)$ in the single $2 \mathrm{~g}$ dose group while no relapse occurred in the multiple dose group. This frequency of clinical relapse in the patients treated with single dose tetracycline was not statistically significant. Larger studies will be required to determine whether the single dose is as effective as the multiple dose when treating relapses.

The duration of excretion of Vibrio cholerae after single dose tetracycline therapy, however, is not as effectively shortened as with multiple dose tetracycline therapy. It is not yet clear whether these asymptomatic culture positive patients represent a significant public health risk to the community, as symptomatic cholera patients only represent a small fraction of the total number of people infected with and excreting Vibrio cholerae during an epidemic.

In a previous study it was shown that in clinical cholera, the duration of antibiotic therapy appeared to be more important than the amount given. ${ }^{2}$ In this study, we have also observed that merely increasing in the dose of tetracycline does not exhance its therapeutic efficacies.

The primary and life saving treatment for clinical cholera remains rehydration and maintenance of continuing losses by oral rehydration or intravenous fluid. Treatment of adult patients with single dose $1 \mathrm{~g}$ or $2 \mathrm{~g}$ tetracycline in addition to careful attention to rehydration should give satisfactory results especially where supervision of patients is minimal such as outpatient clinics and rehydration centres and when busy physicians can ensure that the patient has taken his full course of therapy and does not have to rely on patients compliance. Another advantage of single $1 \mathrm{~g}$ dose therapy is that only half the amount of tetracycline $(1 \mathrm{~g})$ is used compared with multiple dose therapy. Clinical relapses were observed, however, in $7 \%$ cases with single dose therapy. Therefore multiple dose therapy is preferable but single dose treatment might be used in emergencies or when patients with relapses have access to care.

This research was supported by the International Centre for Diarrhoeal Disease Research, Bangladesh (ICDDR,B). ICDDR,B is supported by countries and agencies which share its concern about the impact of diarrhoeal diseases on the developing world. I also would like to thank the staff of the Clinical Research Unit for their excellent help and gratefully acknowledge the invaluable suggestions and assistance of Dr Thomas Butler in preparation of this manuscript and Mr Meer Md Ramzan Ali for skilled secretarial work.

\section{References}

1 Carpenter CCJ, Barua D, Criag K, et al. Clinical studies in Asiatic cholera. IV. Antibiotic therapy in cholera. Bull Johns Hopkins Hosp 1966; 118: 216-29.

2 Lindenbaum J, Greenough WB III, Islam MR. Antibiotic therapy of cholera. Bull WHO 1967; 36: 871-83.

3 Wallace CK, Anderson PN, Brown TC, et al. Optimal antibiotic therapy in cholera. Bull WHO 1968; 39: 239-45.

4 Sack DA, Islam S, Rabbani H, Islam A. Single-dose doxycycline for cholera. Antimicrob Agents Chemother 1978; 14: 462-4.

5 Benenson AS, Islam MR, Greenough WB II. Rapid identification of Vibrio cholerae by darkfield microscopy. Bull WHO 1964; 30: 827-31.

6 World Health Organization. Diarrhoeal diseases control programme. A manual for the treatment of acute diarrhoea. Geneva: WHO, 1980. 25 P. (WHO/CDD/ SER/80.2).

7 Monsur KA. Bacteriological diagnosis of cholera under field conditions. Bull WHO 1963; 28: 387-9.

8 Glass RI, Huq I, Alim ARMA, M Yunus M. Emergence of multiple antibiotic-resistant Vibrio cholerae in Bangladesh. J Infect Dis 1980; 142: 939-42.

9 Towner KH, Pearson NJ, Mhalu FS, O’Grady F. Resistance to antimicrobial agents of Vibrio cholerae EL Tor strains isolated during the fourth cholera epidemic in the United Republic of Tanzania. Bull WHO 1980; 58: 747-51.

10 Woodward WE, Mosley WH. The spectrum of cholera in rural Bangladesh. II. Comparison of El Tor Ogawa and classical Inaba infection. Am J Epidemiol 1972; 96: 342-51. 